\title{
Research on 6-Carboxyl Chitin Promotes the Role of Cytokine Secretion
}

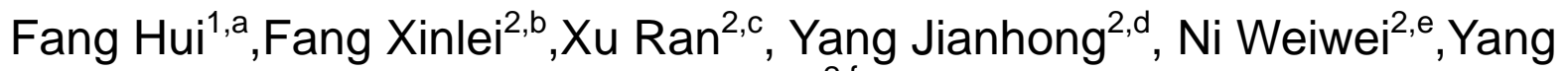 \\ Yan $^{\star 2, f}$
}

1.Changzhou Water\&Wood Environmental Protection Technology Corporation,Changzhou 213016,China;

2. School of Environment\&Safety Engineering, Changzhou University, Changzhou 213016,China

a513798085@qq.com,bczfxinlei@163.com,c124973197@qq.com,

dyangshenyang3@163.com ,eniweiweio@qq.com, ${ }^{*}$ eyy129129@163.com

Keywords: 6-carboxyl of chitin, phagocytes, active, secretions

\begin{abstract}
By enzyme-linked immunosorbent ELISA experiments, Determination of human skin fibroblasts (HSF) and macrophages (white blood cells by monocytes in THP 1) induced by PDGF after 6-carboxyl chitin stimulation, TGF-beta, and the expression of bFGF. The results showed that 6-carboxyl chitin can promote the secretion of macrophage PDGF, its activity is influenced by 6-carboxyl chatoyant molecular weight.
\end{abstract}

\section{Introduction}

In the process of wound healing factors play an important role. Such as PDGF (platelets become part of a growth factor). It can by the platelets, macrophages and endothelial cells and fibroblasts secrete, the active factor of the PDGF can adjust some necessary for tissue repair ${ }^{[1]}$.In addition, it can also promote dermal reconstruction, fibroblast proliferation, collagen, and protein synthesis, promote endothelial migration and the formation of blood vessels, stimulate monocytes/macrophages to secrete growth factors such as the TGF-beta ${ }^{[2,3]}$. Basic fibroblast growth factor (bFGF) in the formation of new blood vessels, and granulation tissue plays a key role. In addition, it can activate the activity of some enzymes, and stimulate the blood vessels, and the migration of endothelial cells and division ${ }^{[4]}$.

By enzyme-linked immunosorbent ELISA experiments, the determination of the human skin fibroblasts (HSF) and macrophages (white blood cells by monocytes in THP 1) induced by PDGF after 6-carboxyl chitin stimulation, TGF-beta, and the expression of bFGF.

\section{The experiment part}

\section{Growth factor concentration}

The spread THP 1 monotype in 24 orifices, the density of 5x105/hole, 48h after 20ng/mL PMA induced into macrophages. The HSF shop is in the heart of the orifice plate, density of 5x105/hole, plus $6-0.1 \mathrm{mg} / \mathrm{mL}$ carboxyl chitin stimulus $12 \mathrm{~h}$, respectively after $24 \mathrm{~h}$ and $72 \mathrm{~h}$ supernatant on collection. This phase medium contains only $5 \%$ of the fetal serum niu niu. According to ELISA kit instructions after the calculation of growth factor concentration in the supernatant.

\section{Induction of macrophages}

Take a certain number of THP 1 cells in cell culture bottles, join containing 20ng/mL of PMA, 10\% serum medium, gently blending quickly after rapid cell ceiling to 24 orifice. In the cell culture box, $5 \% \mathrm{CO}_{2}, 37$ oc incubation 48h. Done induced cells as shown in fig1b (150), one of the most cells have been suspended state to stick wall and cells grow pseudopodia, and most of the cells had pasted wall can be clearly seen under a microscope. In the control group (fig1a), however, saw the 
most THP 1 cells suspended in the medium does not focus, more blurred. These two photos showed the obvious THP 1 induced the difference between before and after, and the literature reports, after PMA stimulation into THP 1 stick wall shows that the cells have been induced into macrophages after $^{[5]}$.
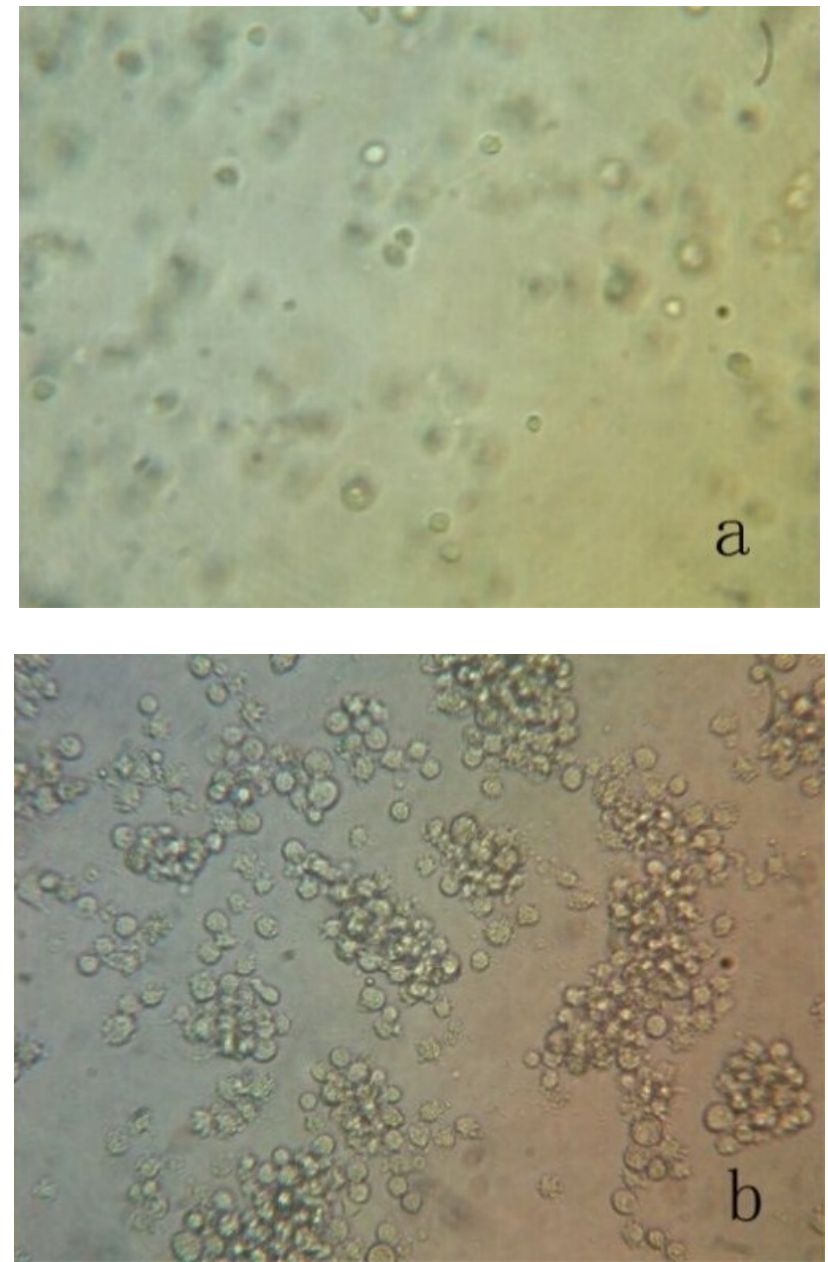

Fig.1 Mononuclear leukocyte THP 1 (a), after PMA induced the mononuclear leukocytes THP 1/ macrophages (b)

\section{Macrophages secrete factors}

Macrophages play an important regulating role in wound healing, research has showed that inhibition of the wound of the activities of macrophages in the wound healing obviously slows down, the role of macrophages in wound healing are mainly; Phagocytosis, secreted collagen as well as the secretion of various growth factors such as PDGF, TGF-beta ${ }^{[3]}$.In order to examine 6-carboxyl of chitin, the function of macrophage macrophages after dosing stimulus by ELISA experiments of PDGF and TGF-beta secretions were studied. 


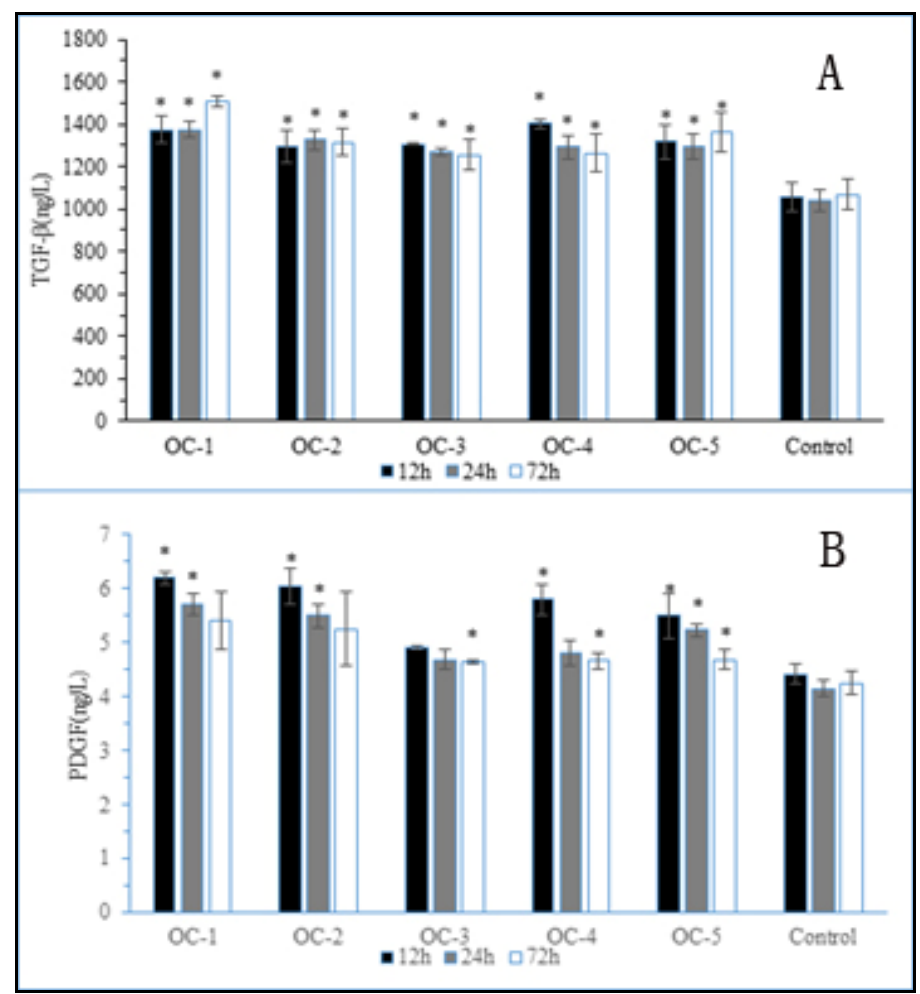

Fig.2 $100 \mathrm{ug} / \mathrm{mL}$ different molecular weight 6-carboxyl chitin stimulus $12 \mathrm{~h}, 24 \mathrm{~h}$ and $72 \mathrm{~h}$ after the macrophages in the supernatant TGF-beta (A) and PDGF (B) the content of (* indicates significant differences $\mathrm{P}<0.05$ )

Fig.2A shows the macrophages by joining $100 \mathrm{ug} / \mathrm{mL}$ different molecular weight 6-carboxyl chitin stimulus $12 \mathrm{~h}, 24 \mathrm{~h}$ and $72 \mathrm{~h}$ after the transforming growth factor in the supernatant TGF-the content of beta. Different molecular weight of 6-the difference between carboxyl chatoyant group and is not very big, and there was no significant difference. Low molecular weight of 6-carboxyl chitin group at OC-1 $72 \mathrm{~h}$, its that the supernatant fluid levels of transforming growth factor (TGF-beta) up to 1507ng/L category levels are higher than all the others, and the content of control group only between 1039-1067ng/L. Shows that 6-carboxyl chatoyant promoted the secretion of macrophage TGF-beta, and the influence of the expression of its molecular weight is not very big.

Fig.2B shows the join 6-carboxyl chitin stimulates macrophage in the supernatant after the content of platelet-derived growth factor (PDGF). Can see from fig.2 b and add 6-carboxyl chitin stimulate macrophages after that the supernatant fluid of platelet-derived growth factor (PDGF) content is higher than the normal control group. And with the increase of time by 6-the content of carboxyl chatoyant stimulation group increased with the increase of time, this may be because the secretion of PDGF only within $12 \mathrm{~h}$ in front and with the increase of time PDGF could be absorbed by the cells. In addition, with growth factors secreted by macrophages, the content of platelet-derived growth factor (PDGF) with molecular weight rises to decline to rise again. Low molecular weight such as OC-1, 6-carboxyl chitin OC-2 to promote macrophage the exudation of platelet-derived growth factor (PDGF). From the above results, the 6-carboxyl chitin can promote the secretion of macrophage PDGF, its activity is influenced by 6-carboxyl chatoyant molecular weight.

\section{Conclusions}

(1) After PMA stimulation THP 1 cells become adherent growth, THP-1 indicates that the cells have been inducted into macrophages

(2) 6-carboxyl chitin can promote the secretion of macrophage PDGF, its activity is influenced by 6-carboxyl chatoyant molecular weight. 


\section{Acknowledgements}

This work was financially supported by Changzhou city science and technology support program (social development, Grant No.CE20125036).

\section{References:}

[1] C.Heldin, B.Westermark.Cell Regulation.Forum Vol.1(1990),p.555-566

[2]D.Shreiber,P.Enever,R.Tranquillo.American Society of Mechanical Engineers. Forum Vol.48(1990),p.183-184

[3]N.Kubota,N.Tatsumoto,T.Sano,et al.Carbohydrate Research. Forum Vol.4(2000),p. 268-274

[4]G.Pierce,T.Mustoe,J.Lingelbach,et al.Cell Biology.Forum Vol.86(1989), p.2229-2233

[5]R.Montesano,J.Vassalli,A.Bairdt,et al.Cell Biology. Forum Vol.83(1986), p.7297-9301

[6]J.Huang,S.Wu,X.Xie,etal.European Journal of Pharmacology. Forum Vol.670 (2011),p.304-310 